\title{
Analysis on $\psi$-Hilfer Fractional Impulsive Differential Equations
}

\author{
Kulandhaivel Karthikeyan ${ }^{1} \mathbb{D}$, Panjaiyan Karthikeyan ${ }^{2}{ }^{\oplus}$, Dimplekumar N. Chalishajar ${ }^{3, *}$, \\ Duraisamy Senthil Raja ${ }^{4}\left(\mathbb{C}\right.$ and Ponnusamy Sundararajan ${ }^{5}(\mathbb{C}$
}

1 Department of Mathematics, KPR Institute of Engineering and Technology, Coimbatore 641 407, Tamil Nadu, India; karthikeyan.k@kpriet.ac.in

2 Department of Mathematics, Sri Vasavi College, Erode 638 316, Tamil Nadu, India; drpk@srivasavi.ac.in

3 Department of Applied Mathematics, Virginia Military Institute, 435 Mallory Hall, Lexington, VA 24450, USA

4 Department of Mathematics, K.S.Rangasamy College of Technology,

Tiruchengode 637 215, Tamil Nadu, India; senthilrajad@ksrct.ac.in

5 Department of Mathematics, Arinagar Anna Government Arts College,

Namakkal 637 002, Tamil Nadu, India; p.sundararajan@aagacnkl.edu.in

* Correspondence: Chalishajardn@vmi.edu

Citation: Karthikeyan, K.;

Karthikeyan, P.; Chalishajar, D.N.;

Raja, D.S.; Sundararajan, P. Analysis on $\psi$-Hilfer Fractional Impulsive Differential Equations. Symmetry 2021, 13, 1895. https://doi.org/ $10.3390 /$ sym 13101895

Academic Editors: Włodzimierz Fechner and Jacek Chudziak

Received: 17 July 2021

Accepted: 2 October 2021

Published: 8 October 2021

Publisher's Note: MDPI stays neutral with regard to jurisdictional claims in published maps and institutional affiliations.

\begin{abstract}
In this manuscript, we establish the existence of results of fractional impulsive differential equations involving $\psi$-Hilfer fractional derivative and almost sectorial operators using Schauder fixed-point theorem. We discuss two cases, if the associated semigroup is compact and noncompact, respectively. We consider here the higher-dimensional system of integral equations. We present herewith new theoretical results, structural investigations, and new models and approaches. Some special cases of the results are discussed as well. Due to the nature of measurement of noncompactness theory, there exists a strong relationship between the sectorial operator and symmetry. When working on either of the concepts, it can be applied to the other one as well. Finally, a case study is presented to demonstrate the major theory.
\end{abstract}

Keywords: $\psi$-Hilfer fractional derivative; mild solutions; impulsive conditions; almost sectorial operators; measure of noncompactness

\section{Introduction}

Hilfer [1] started the Hilfer fractional derivative, an extended Riemann-Liouville fractional derivative that interpolates Caputo fractional derivatives and Riemann-Liouville fractional derivatives. Theoretical simulations of dielectric relaxation in glass-forming materials revealed the existence of this operator. Hilfer et al. citeH3 first presented linear differential equations with the new Hilfer fractional derivative and solved such generalised fractional differential equations using operational calculus.

In the conventional equation for exponential relaxation, the infinitesimal generator of time evolution is substituted by the infinitesimal generator of composite fractional translations [2]. Translation and fractional time evolution are combined to form composite fractional translations. Also solved is the fractional differential equation for composite fractional relaxation. The obtained dynamical susceptibility is utilised to fit data from glycerol's broad-band dielectric spectroscopy. At high frequencies in the imaginary part, the composite fractional susceptibility function might show an asymmetric relaxation peak and an excess wing. Despite this, it only has a single stretch.

We consider the following fractional impulsive differential equations involving $\psi$ Hilfer fractional derivative:

$$
\begin{aligned}
\mathfrak{D}^{E, \beta ; \psi} \wp(t)+\mathfrak{A} \wp(t) & =\mathscr{E}(t, \wp(t)) \quad t \in \mathcal{J}^{\prime}=(0, T] \\
\left.\Delta \wp\right|_{t=t_{k}} & =\mathscr{I}_{k}\left(\wp\left(t_{k}^{-}\right)\right), k=1,2,3, \ldots, m, \\
I_{0+}^{(1-\lambda)(1-\beta) ; \psi} \wp(0) & =\wp_{0},
\end{aligned}
$$


where $\mathfrak{D}_{0+}^{£, \beta ; \psi} \psi$ - Hilfer fractional derivative of order $£ \in(0,1)$ and type $\beta \in \mathcal{J}=[0, T] . \mathfrak{A}$ is an almost sectorial operator on Banach space $Y$ with norm $\|\cdot\|, \quad \mathscr{E}: \mathcal{J} \times Y \rightarrow Y$ is well defined on $Y .\left.\Delta \wp\right|_{t=t_{k}}$ denotes the jump of $\wp(t)$ at $t=t_{k}$, i.e., $\left.\Delta \wp\right|_{t=t_{k}}=\wp\left(t_{k}^{+}\right)-\wp\left(t_{k}^{-}\right)$, where $\wp\left(t_{k}^{+}\right)$and $\wp\left(t_{k}^{-}\right)$represent the right and left limits of $u(t)$ at $t=t_{k}$, respectively.

Many approaches are being utilized to investigate and manage differential equations in both fractional and classical orders, as well as their systems, including residual power series, symmetry, spectral, Fourier transform, similarity, and collocation methods. A sectorial operator has a direct connection with the convexity. The concept of convexity of the sectorial operator plays a strong role in the field of mathematical inequalities and mathematical analysis due to the beautiful nature of its classical definition and its algebraic properties. Recently, many authors have explored the close relationship and interrelated work on convexity and symmetry. Due to the strong relationship between them, while working on any one of the concepts it can be applied to the other one as well. The sectorial operator with measure of noncompactness theory has gained a lot of attention in recent years and many generalizations and refinements of existence, uniqueness and stability analysis for inequalities have been found in the literature.

Hilfer [3] on the other hand, introduced a fractional derivative $D^{\gamma, \eta}($.$) having two$ parameters $\eta \in(n-1, n), n \in N$ and $0 \leq \gamma \leq 1$, which in specific gives the RiemannLiouville and the Caputo derivative operators. For detailed advantages of Hilfer derivative, please refer to Section 4 of [4]. In [5], Abdo et al. studied the existence and Ulam-stability results for $\psi$-Hilfer fractional integro-differential equations. In [6], a monotone iterative technique was used to solve initial value problems for nonlinear fractional differential equations with $\psi$-Caputo derivative. The $\psi$-Hilfer fractional derivative differential equations with boundary value problems were discussed by Mali et al. in [7].

In [8], Kucche et al. discussed the nonlinear $\psi$-Hilfer fractional derivative differential equations with initial value problems of the form

$$
\begin{aligned}
& { }^{H} \mathfrak{D}^{£, \beta ; \psi} \wp(t)=\mathscr{F}(t, \wp(t)) \quad 0<£<1,0 \leq \beta \leq 1, \\
& I_{0+}^{(1-\zeta ; \psi)} \wp(a)=\wp_{a} \text {, }
\end{aligned}
$$

where ${ }^{H} \mathfrak{D}^{£, \beta ; \psi}$ is the $\psi$-Hilfer derivative of order $£$ and $\mathscr{F}$ is an appropriate function.

In [9], Sousa et al. studied the stability of the modified impulsive fractional differential equations of the form

$$
\begin{aligned}
H_{\mathfrak{D}^{£, \beta ; \psi} \wp(t)}=\mathscr{F}(t, \wp(t)) \quad 0<£<1,0 \leq \beta \leq 1, \\
\wp(t)=g_{i}\left(t, \wp\left(t_{i}^{+}\right),\right.
\end{aligned}
$$

where ${ }^{H} \mathfrak{D}^{£, \beta ;} ;$ - is the $\psi$-Hilfer fractional derivative and $\mathscr{F}, g$-is continuous. We recommend readers to publications of Sousa and Oliveira $[10,11]$ for the essential properties of $\psi$-Hilfer fractional derivative and the basic theory of fractional differential equations involving $\psi$-Hilfer fractional derivative.

The work is organized as follows: in Section 2, we discuss the Hilfer derivative, almost sectorial operators, measure of noncompactness, and mild solutions of Equations (1)-(3), as well as some basic definitions and lemmas. In Section 3, we go over the major analysis of mild solutions to Equations (1)-(3). We cover two scenarios where the related semigroup is compact and noncompact in Sections 4 and 5, respectively. Finally, an abstract application for the primary conclusion is discussed.

The following section describes the supporting results of the given problem which generalizes the results in [12]:. 


\section{Preliminaries}

Definition 1 ([13]). The $\psi$-Hilfer fractional derivative of the function $f$ is given by

$$
\mathfrak{D}^{v, \S ; \psi} f(t)=\mathfrak{I}^{\S(1-\beta) ; \psi}\left(\frac{1}{\psi^{\prime}(t)} \frac{d}{d t}\right) \mathfrak{I}^{(1-\S)(1-\beta) \psi} f(t) .
$$

\section{Measure of Noncompactness:}

Let $\digamma$ e the bounded set in Banach space $Y$. We consider the Hausdorff measure of noncompactness [12] as follows:

$$
\aleph(\digamma)=\inf \left\{\zeta>0 \text { that is } \digamma \subset \bigcup_{j=1}^{m} B_{\zeta}\left(x_{j}\right) \quad \text { where } \quad x_{j} \in Y, m \in N\right\} \text {, }
$$

where $B_{\zeta}\left(x_{j}\right)$ is a ball centered at $x_{j}, j=1,2, \ldots, m$ with a radius $\zeta \leq \mathfrak{F}$. On a bounded set $\mathcal{B} \subset Y$, the Kuratowski measure of noncompactness $\Phi$ is considered as follows:

$$
\Phi(\digamma)=\inf \left\{\mathfrak{F}>0 \quad \text { implies } \quad \digamma \subset \bigcup_{j=1}^{m} M_{j} \quad \text { also } \quad \operatorname{diam}\left(M_{j}\right) \leq \mathfrak{F}\right\},
$$

where $M_{j}$ has a diameter defined by $\operatorname{diam}\left(M_{j}\right)=\sup \left\{\|x-y\|: x, y \in M_{j}\right\}, j=1,2, \ldots, m$. The following properties are useful for further investigation:

1. $\digamma_{1} \subset \digamma_{2}$ implies $\aleph\left(\digamma_{1}\right) \leq \aleph\left(\digamma_{2}\right)$ where $\digamma_{1}, \digamma_{2}$ are constrained subsets of $Y$.

2. $\aleph(\digamma)=0$ iff $\digamma$ is quite small in size in $Y$.

3. $\aleph(\{z\} \cup \digamma)=\aleph(\digamma)$ for everyone $z \in Y, \digamma \subseteq Y$.

4. $\aleph\left(\digamma_{1} \cup \digamma_{2}\right) \leq \max \left\{\aleph\left(\digamma_{1}\right), \aleph\left(\digamma_{2}\right)\right\}$.

5. $\aleph\left(\digamma_{1}+\digamma_{2}\right) \leq \aleph\left(\digamma_{1}\right)+\aleph\left(\digamma_{2}\right)$.

6. $\aleph(r \digamma) \leq|r| \aleph(\digamma)$ for $r \in R$.

Almost sectorial opertors: Let $0<\mu<\pi$ and $-1<\S<0$. We define $S_{\mu}^{0}=\{v \in$ $\mathbb{C} \backslash\{0\}$ such that $|\arg v|<\mu\}$ and its closure by $S_{\mu}$, where $S_{\mu}=\{v \in \mathbb{C} \backslash\{0\}|\arg v|<$ $\mu\} \bigcup\{0\}$.

Proposition 1 ([14]). Let $\mathfrak{A} \in \odot \bigodot_{\omega}^{\S}$ for $-1<\S<0$ and $0<\omega<\frac{\pi}{2}$. Then the below properties hold.

1. $\quad \mathfrak{H}(t)$ is analytic and $\frac{d^{n}}{d t^{n}} \mathfrak{H}(t)=\left(-\mathfrak{A}^{n} \mathfrak{H}(t)\left(t \in S_{\frac{\pi}{2}}^{0}\right)\right.$.

2. $\quad \mathfrak{H}(t+s)=\mathfrak{H}(t) \mathfrak{H}(s) \quad \forall t, s \in S_{\frac{\pi}{2}}^{0}$.

3. $\|\mathfrak{H}(t)\|_{L(\mathscr{Y})} \leq C_{0} t^{-\S-1}(t>0)$, where $C_{0}=C_{0}(\S)>0$ is a constant.

4. Let $\sum_{\mathfrak{H}}=\left\{x \in \mathscr{Y}: \lim _{t \rightarrow 0_{+}} \mathfrak{H}(t) x=x\right\}$. Additionally, $\mathfrak{D}\left(\mathfrak{A}^{\theta}\right) \subset \sum_{\mathfrak{Q}}$ if $\theta>1+\S$.

5. $\mathscr{R}(r,-\mathfrak{A})=\int_{0}^{\infty} e^{-r s} \mathfrak{H}(s) d s, r \in \mathbb{C}$ with $\operatorname{Re}(r)>0$.

It is observed that $\mathfrak{M}$ is a Wright-type function [15] such that

$$
\mathfrak{M}_{£}(\theta)=\sum_{n \in \mathbb{N}} \frac{(-\theta)^{n-1}}{\Gamma(1-£ n)(n-1) !}, \quad \theta \in \mathbb{C} .
$$

For $-1<\sigma<\infty, r>0$,

(A1) $\mathfrak{M}_{£}(\theta) \geq 0, t>0$.

(A2) $\int_{0}^{\infty} \theta^{\sigma} \mathfrak{M}_{£} d \theta=\frac{\Gamma(1+\sigma)}{\Gamma(1+£ \sigma)}$

(A3) $\int_{0}^{\infty} \frac{£}{\theta^{£+1}} e^{-r \theta} \mathfrak{M}_{£}\left(\frac{1}{\theta^{£}}\right) d \theta=e^{-r^{£}}$.

We define $\left.\left\{\mathfrak{S}_{\ell}(t)\right\}\right|_{t \in S_{\frac{\pi}{2}-w}^{0}},\left.\left\{\mathfrak{Q}_{\ell}(t)\right\}\right|_{t \in S_{\frac{\pi}{2}-w}^{0}}$ by 


$$
\begin{gathered}
\mathfrak{S}_{£}(t)=\int_{0}^{\infty} \mathfrak{M}_{£}(\zeta) \varrho\left(t^{£} \zeta\right) d \zeta, \\
\mathfrak{Q}_{£}(t)=\int_{0}^{\infty} £ \zeta \mathfrak{M}_{£}(\zeta) \varrho\left(t^{£} \zeta\right) d \zeta .
\end{gathered}
$$

Proposition 2. We define $\mathfrak{B}_{r}(\mathcal{J})=\{y \in \mathscr{Y}=C(\mathcal{J}, Y)$ such that $\|y\| \leq r\}$ and $\mathfrak{B}_{r}^{\mathscr{Y}}\left(\mathcal{J}^{\prime}\right)=$ $\left\{\wp \in \mathscr{Y}\right.$ such that $\|\wp\|_{\mathscr{Y}} \leq r$, where $\left.\|\wp\|_{\mathscr{Y}}=\sup _{t \in J}\|\wp(t)\|\right\}$.

Proposition 3. We define an operator $\mathfrak{F}: \mathfrak{B}_{r}(\mathcal{J}) \rightarrow \mathfrak{B}_{r}(\mathcal{J})$ by

$$
(\mathfrak{F} y)(t)=\left\{\begin{array}{l}
0 \text { if } t=0, \\
t^{(1+£ \mu)(1-\beta)} \mathcal{F} \wp(t), t \in(0, T],
\end{array}\right.
$$

where $\mathcal{F}: \mathfrak{B}_{r}\left(\mathcal{J}^{\prime}\right) \rightarrow \mathfrak{B}_{r}\left(\mathcal{J}^{\prime}\right), \mathcal{J}^{\prime}=(0, T]$ and $\wp(t)=t^{-(1+£ \mu)(1-\beta)} y(t)$ for $y \in \mathfrak{B}_{r}(\mathcal{J})$.

Then, to show the key results, we assume following conditions:

Hypothesis 1 (H1). For $t \in \mathcal{J}, \mathscr{E}(t,):. Y \rightarrow Y$ is continuous function as well as each $\wp \in \mathfrak{C}(\mathcal{J}, Y), \mathscr{E}(., \wp): \mathcal{J} \rightarrow Y$ is strongly measurable.

Hypothesis 2 (H2). $\exists k \in L^{1}\left(\mathcal{J}, \mathbb{R}^{+}\right)$satisfying

$$
I_{0+}^{-£ \S} k \in \mathfrak{C}(\mathcal{J}, \mathbb{R}), \lim (\psi(t))^{(1+£ \S)(1-\beta)} I_{0+}^{-£ \S} k(t)=0 .
$$

Hypothesis 3 (H3).

$$
\begin{aligned}
& \sup _{[0, T]}\left[(\psi(t))^{(1+£ \S)(1-\beta)}\left\|\psi_{0}^{(1-£)(\beta-1)}\right\|+(\psi(t))^{(1+£ \S)(1-\beta)} \int_{0}^{t} \psi^{\prime}(r)[\psi(t)-\psi(r)]^{-£ \S-1} k(r) d r\right] \leq r, \\
& \text { for } r>0 .
\end{aligned}
$$

Hypothesis 4 (H4). $\exists$ constants $\beta_{k}$ such that $\left\|\mathscr{I}_{k}(\wp)\right\| \leq \omega_{k}, k=1,2, \ldots, m$ for each $\wp \in Y$.

Definition 2. By a mild solution of the Cauchy Problem (1)-(3) we mean a function $\wp \in \mathfrak{C}(\mathcal{J}, Y)$ that satisfies the following integral condition:

$$
\begin{aligned}
\wp(t)= & \left(\psi_{0}\right)^{(1-\ell)(\beta-1)} \wp_{0}+\frac{1}{\Gamma(£)} \int_{0}^{t} \psi^{\prime}(r) \mathfrak{K}_{\epsilon}(\psi(t)-\psi(r)) \mathscr{E}(r, \wp(r)) d r \\
& +\sum_{0<t_{k}<t} \psi_{0}^{£, \beta}\left(\psi\left(t_{2}\right)-\psi\left(t_{k}\right)\right) \mathscr{I}_{k}\left(\wp\left(t_{k}^{-}\right)\right), \quad t \in \mathcal{J},
\end{aligned}
$$

where

$$
\psi_{0}^{(1-£)(\beta-1)}=\frac{(\psi(t)-\psi(0))^{(1-£)(\beta-1)}}{\Gamma(v(1-£)+£}, \mathfrak{K}_{£}=\psi(t)^{£-1} \mathfrak{Q}_{£} \psi(t)
$$

\section{Main Results}

Theorem 1. Let $\mathfrak{A} \in \odot \bigodot_{\omega}^{\S}$ for $-1<\S<0$ and $0<\omega<\frac{\pi}{2}$. Assuming $(H 1)-(H 4)$ are satisfied, the operators $\left\{\mathfrak{F} y: y \in \mathfrak{B}_{r}(\mathcal{J})\right\}$ is equicontinuous as long as $\wp_{0} \in \mathcal{D}\left(\mathfrak{A}^{\theta}\right)$ with $\theta>1+\S$. 
Proof. We have

$$
\begin{aligned}
\left\|\mathfrak{F} y\left(t_{2}\right)-\mathfrak{F} y(0)\right\|= & \| t_{2}^{(1+£ \mu)(1-\beta)}\left(\left(\psi_{0}\left(t_{2}\right)\right)^{(1-£)(\beta-1)} \wp_{0}+\frac{1}{\Gamma(£)} \int_{0}^{t_{2}} \psi^{\prime}(r)\left(\psi\left(t_{2}\right)-\psi(r)\right)^{£-1}\right. \\
& \left.\mathfrak{Q}_{£}\left(\psi\left(t_{2}\right)-\psi(r)\right) \mathscr{E}(r, \wp(r)) d r+\sum_{0<t_{k}<t_{2}} \psi_{0}^{£, \beta}\left(\psi\left(t_{2}\right)-\psi\left(t_{k}\right)\right) \mathscr{I}_{k}\left(\wp\left(t_{k}^{-}\right)\right)\right) \|, \\
& \leq\left\|t_{2}^{(1+£ \mu)(1-\beta)}\left(\psi_{0}\left(t_{2}\right)\right)^{(1-£)(\beta-1)} \wp_{0}\right\| \\
& +\frac{1}{\Gamma(£)}\left\|t_{2}^{(1+£ \mu)(1-\beta)} \int_{0}^{t_{2}} \psi^{\prime}(r)\left(\psi\left(t_{2}\right)-\psi(r)\right)^{£-1} \mathfrak{Q}_{£}\left(\psi\left(t_{2}\right)-\psi(r)\right) \mathscr{E}(r, \wp(r)) d r\right\| \\
& +\left\|t_{2}^{(1+£ \mu)(1-\beta)} \sum_{0<t_{k}<t_{2}} \psi_{0}^{£, \beta}\left(\psi\left(t_{2}\right)-\psi\left(t_{k}\right)\right) \mathscr{I}_{k}\left(\wp\left(t_{k}^{-}\right)\right)\right\| \\
& \rightarrow 0, \text { as } t_{2} \rightarrow 0 .
\end{aligned}
$$

Now, let $0<t_{1}<t_{2} \leq T$,

$$
\begin{aligned}
& \left\|\mathfrak{F} y\left(t_{2}\right)-\mathfrak{F} y\left(t_{1}\right)\right\| \leq \| t_{2}^{(1+£ \mu)(1-\beta)}\left(\left(\psi_{0}\left(t_{2}\right)\right)^{(1-£)(\beta-1)} \wp_{0}-t_{1}^{(1+£ \mu)(1-\beta)}\left(\left(\psi_{0}\left(t_{1}\right)\right)^{(1-£)(\beta-1)} \wp_{0} \|\right.\right. \\
& +\| t_{2}^{(1+£ \mu)(1-\beta)} \int_{0}^{t_{2}} \psi^{\prime}(r)\left(\psi\left(t_{2}\right)-\psi(r)\right)^{£-1} \mathfrak{Q}_{£}\left(\psi\left(t_{2}\right)-\psi(r)\right) \mathscr{E}(r, \wp(r)) d r \\
& -t_{1}^{(1+£ \mu)(1-\beta)} \int_{0}^{t_{1}} \psi^{\prime}(r)\left(\psi\left(t_{1}\right)-\psi(r)\right)^{£-1} \mathfrak{Q}_{£}\left(\psi\left(t_{1}\right)-\psi(r)\right) \mathscr{E}(r, \wp(r)) d r \| \\
& +\| t_{2}^{(1+f \mu)(1-\beta)} \sum_{0<t_{k}<t_{2}} \psi_{0}^{£, \beta}\left(\psi\left(t_{2}\right)-\psi\left(t_{k}\right)\right) \mathscr{I}_{k}\left(\wp\left(t_{k}^{-}\right)\right) \\
& -t_{1}^{(1+£ \mu)(1-\beta)} \sum_{0<t_{k}<t_{1}} \psi_{0}^{£, \beta}\left(\psi\left(t_{2}\right)-\psi\left(t_{k}\right)\right) \mathscr{I}_{k}\left(\wp\left(t_{k}^{-}\right)\right) \| \text {. }
\end{aligned}
$$

Using the triangle inequality, we have

$$
\begin{aligned}
& \left\|\mathfrak{F} y\left(t_{2}\right)-\mathfrak{F} y\left(t_{1}\right)\right\| \leq \| t_{2}^{(1+£ \mu)(1-\beta)}\left(\left(\psi_{0}\left(t_{2}\right)\right)^{(1-£)(\beta-1)} \wp_{0}-t_{1}^{(1+£ \mu)(1-\beta)}\left(\left(\psi_{0}\left(t_{1}\right)\right)^{(1-£)(\beta-1)} \wp_{0} \|\right.\right. \\
& +\left\|t_{2}^{(1+£ \mu)(1-\beta)} \int_{t_{1}}^{t_{2}} \psi^{\prime}(r)\left(\psi\left(t_{2}\right)-\psi(r)\right)^{£-1} \mathfrak{Q}_{E}\left(\psi\left(t_{2}\right)-\psi(r)\right) \mathscr{E}(r, \wp(r)) d r\right\| \\
& +\| t_{2}^{(1+£ \mu)(1-\beta)} \int_{0}^{t_{1}} \psi^{\prime}(r)\left(\psi\left(t_{2}\right)-\psi(r)\right)^{£-1} \mathfrak{Q}_{£}\left(\psi\left(t_{2}\right)-\psi(r)\right) \mathscr{E}(r, \wp(r)) d r \\
& -t_{1}^{(1+£ \mu)(1-\beta)} \int_{0}^{t_{1}} \psi^{\prime}(r)\left(\psi\left(t_{1}\right)-\psi(r)\right)^{£-1} \mathfrak{Q}_{\mathcal{E}}\left(\psi\left(t_{2}\right)-\psi(r)\right) \mathscr{E}(r, \wp(r)) d r \| \\
& +\| t_{1}^{(1+£ \mu)(1-\beta)} \int_{0}^{t_{1}} \psi^{\prime}(r)\left(\psi\left(t_{1}\right)-\psi(r)\right)^{£-1} \mathfrak{Q}_{\ell}\left(\psi\left(t_{2}\right)-\psi(r)\right) \mathscr{E}(r, \wp(r)) d r \\
& -t_{1}^{(1+£ \mu)(1-\beta)} \int_{0}^{t_{1}} \psi^{\prime}(r)\left(\psi\left(t_{1}\right)-\psi(r)\right)^{£-1} \mathfrak{Q}_{\mathcal{E}}\left(\psi\left(t_{2}\right)-\psi(r)\right) \mathscr{E}(r, \wp(r)) d r \| \\
& +\| t_{2}^{(1+£ \mu)(1-\beta)} \sum_{0<t_{k}<t_{2}} \psi_{0}^{£, \beta}\left(\psi\left(t_{2}\right)-\psi\left(t_{k}\right)\right) \mathscr{I}_{k}\left(\wp\left(t_{k}^{-}\right)\right) \\
& -t_{1}^{(1+£ \mu)(1-\beta)} \sum_{0<t_{k}<t_{1}} \psi_{0}^{£, \beta}\left(\psi\left(t_{2}\right)-\psi\left(t_{k}\right)\right) \mathscr{I}_{k}\left(\wp\left(t_{k}^{-}\right)\right) \| \\
& =\mathfrak{I}_{1}+\mathfrak{I}_{2}+\mathfrak{I}_{3}+\mathfrak{I}_{4}+\mathfrak{I}_{5} .
\end{aligned}
$$

Using $\mathfrak{S}_{£, \beta}(t)$, we get $\mathfrak{I}_{1} \rightarrow 0$ as $t_{2} \rightarrow t_{1}$.

Additionally,

$$
\begin{aligned}
\mathfrak{I}_{2} & \leq \mathfrak{C}_{p} \psi_{0} t_{2}^{(1+£ \S)(1-\beta)} \int_{t_{1}}^{t_{2}} \psi^{\prime}(r)\left(\psi\left(t_{1}\right)-\psi(r)\right)^{-£ \S-1} \kappa(r) d r \\
& \leq \mathfrak{C}_{p} \int_{0}^{t_{1}}\left|\psi_{0} t_{1}^{(1+£ \S)(1-\beta)} \psi^{\prime}(r)\left(\psi\left(t_{1}\right)-\psi(r)\right)^{-£ \S-1}-t_{2}^{(1+£ \S)(1-\beta)} \psi^{\prime}(r)\left(\psi\left(t_{2}\right)-\psi(r)\right)^{-£ \S-1}\right| \kappa(r) d r .
\end{aligned}
$$


Then $\mathfrak{I}_{2} \rightarrow 0$ as $t_{2} \rightarrow t_{1}$, by using (H2). Since

$$
\begin{aligned}
\mathfrak{I}_{3} \leq & \mathfrak{C}_{p} \int_{0}^{t_{1}} \psi^{\prime}(r)\left(\psi\left(t_{2}\right)-\psi(r)\right)^{-\ell-£ \S} \\
& \left|t_{2}^{(1+£ \S)(1-\beta)} \psi^{\prime}(r)\left(\psi\left(t_{2}\right)-\psi(r)\right)^{£-1}-t_{1}^{(1+£ \S)(1-\beta)} \psi^{\prime}(r)\left(\psi\left(t_{1}\right)-\psi(r)\right)^{\ell-1}\right| \kappa(r) d r .
\end{aligned}
$$

For $\mathfrak{F}>0$, we have

$$
\begin{aligned}
& \mathfrak{I}_{4}=\leq t_{1}^{(1+£ \S)(1-\beta)+£(1+\S)} \int_{0}^{t_{1}} \psi^{\prime}(r)\left(\psi\left(t_{1}\right)-\psi(r)\right)^{-£ \S-1} \mathcal{K}(r) d r \sup _{s \in\left[0, t_{1}-\mathfrak{F}\right]} \\
& \left\|\mathfrak{Q}_{E}\left(\psi\left(t_{2}\right)-\psi(r)\right)-\mathfrak{Q}_{E}\left(\psi\left(t_{1}\right)-\psi(r)\right)\right\|_{L(X)} \\
& +2 \mathfrak{C}_{p} \int_{t_{1}-\mathfrak{F}}^{t_{1}} t_{1}^{(1+£ \S)(1-\beta)} \psi^{\prime}(r)\left(\psi\left(t_{1}\right)-\psi(r)\right)^{-£ \S-1} \mathcal{K}(r) d r .
\end{aligned}
$$

Clearly, since the strongly continuous of $\mathfrak{S}_{£, \beta}(t)$, we obtain

$$
\begin{array}{r}
\Im_{5}=\| t_{2}^{(1+£ \mu) 1-\beta} \sum_{0<t_{k}<t_{2}} \psi_{0}^{£, \beta}\left(\psi\left(t_{2}\right)-\psi\left(t_{k}\right)\right) \mathscr{I}_{k}\left(\wp\left(t_{k}^{-}\right)\right) \\
-t_{1}^{(1+£ \mu)(1-\beta)} \sum_{0<t_{k}<t_{1}} \psi_{0}^{£, \beta}\left(\psi\left(t_{2}\right)-\psi\left(t_{k}\right)\right) \mathscr{I}_{k}\left(\wp\left(t_{k}^{-}\right)\right) \| \rightarrow 0 \text { as } t_{2} \rightarrow t_{1} .
\end{array}
$$

$\mathfrak{I}_{5} \rightarrow 0$ as $t_{2} \rightarrow t_{1}$.

Hence $\left\|\mathfrak{F} y\left(t_{2}\right)-\mathfrak{F} y\left(t_{1}\right)\right\| \rightarrow 0$, therefore $\left\{\mathfrak{F} y: y \in \mathfrak{B}_{r}(\mathcal{J})\right\}$ is equicontinuous.

Theorem 2. Let $-1<\S<0$ and $0<\omega<\frac{\pi}{2}$ and $\mathfrak{A} \in \odot_{\omega}^{\S}$. After using (H1)-(H3), the operator $\left\{\mathfrak{F} y: y \in \mathfrak{B}_{r}(\mathcal{J})\right\}$ is both continuous and constrained.

Proof. We confirm that $\mathfrak{F}$ maps $\mathfrak{B}_{r}(\mathcal{J})$. Now we have $\wp \in \mathfrak{B}_{r}^{\mathscr{Y}}\left(\mathcal{J}^{\prime}\right)$.

$$
\|\mathfrak{F}\| \leq\left\|t^{(1+£ \mathcal{S})(1-\beta)} \psi_{0}^{£, \beta ; \psi}(t) \wp_{0}\right\|+t^{(1+£ \mathcal{S})(1-\beta)}\left\|\int_{0}^{t} \psi^{\prime}(r)(\psi(t)-\psi(r))^{\ell-1} \mathfrak{Q}_{£}(\psi(t)-\psi(r)) \mathscr{E}(r, \wp(r)) d r\right\| .
$$

From (H2)-(H3), we obtain

$$
\begin{aligned}
\|\mathfrak{F} y(t)\| & \leq t^{(1+£ \S)(1-\beta)}\left\|\mathfrak{S}_{£, \beta}(t) u_{0}\right\|+t^{(1+£ \S)(1-\beta)} \int_{0}^{t} \psi^{\prime}(r)(\psi(t)-\psi(r))^{-£ \S-1} \mathcal{K}(r) d r, \\
& \leq r .
\end{aligned}
$$

Hence $\|\mathfrak{F} y\| \leq r$ for any $y \in \mathcal{B}_{r}(\mathcal{J})$.

From $(\mathrm{H} 1) \&(\mathrm{H} 2)$ to obtain the inequality

$$
\psi^{\prime}(r)(\psi(t)-\psi(r))^{-£ \S-1}\left|\mathscr{E}\left(r, \wp_{n}(r)\right)\right| \leq 2 \psi^{\prime}(r)(\psi(t)-\psi(r))^{-(£ \S)(1-\beta)} \mathcal{K}(r) .
$$

Now to apply [15] (Theorem 4.6.1), we have

$$
\begin{aligned}
& \left\|\mathfrak{F} y_{n}(t)-\mathfrak{F} y(t)\right\| \leq \mathfrak{C}_{p} t^{(1+f \S)(1-\beta)} \int_{0}^{t} \psi^{\prime}(r)(\psi(t)-\psi(r))^{-f \mathcal{S}-1}\left\|\mathscr{E}\left(r, \wp_{n}(r)\right)-\mathscr{E}(r, \wp(r))\right\| d r, \\
& \rightarrow 0 \text { as } n \rightarrow \infty \text {. i.e., } \mathfrak{F} \text { is continuous. }
\end{aligned}
$$

Remark 1. In this section, we have proved the equicontinuity and the continuity of the sectorial operator $\left\{\mathfrak{F} y: y \in \mathfrak{B}_{r}(\mathcal{J})\right\}$. We will be using the continuity result in Section 4 for the compactness of the sectorial operator, and we will be using Arzela-Ascoli theorem which demands 
the equicontinuity and uniform boundedness for the case of noncompactness of the sectorial operator in Section 5.

\section{4. $\Im(t)$ Is Compact}

Theorem 3. Let $-1<\S<0,0<\omega<\frac{\pi}{2}$ and $\mathfrak{A} \in \aleph_{\omega}^{\S}$. If $\mathfrak{T}(t)(t>0)$ is compact and (H1)-(H4) are satisfied, then there is a mild solution of (1)-(3) in $\mathcal{B}_{r}^{\mathcal{Y}}(\mathcal{J})$.

Proof. We are now in position to define $\mathfrak{F}: \mathfrak{B}_{r}(\mathcal{J}) \rightarrow \mathfrak{B}_{r}(\mathcal{J})$ by

$$
(\mathfrak{F} y)(t)=\left(\mathfrak{F}^{1} y\right)(t)+\left(\mathfrak{F}^{2} y\right)(t) .
$$

For $\sigma>0$ and $\zeta \in(0, t)$, we have

$$
\begin{aligned}
\left(\mathfrak{F}_{\zeta, \sigma}^{1} y\right)(t) & =\frac{(\psi(t))^{(1+£ \S)(1-\beta)}}{\Gamma(\beta(1-£))} \int_{\zeta}^{t} \int_{\sigma}^{\infty} \psi^{\prime}(\psi(t)-\psi(r))^{(1-£) \beta-1} r^{£-1} \theta M_{\mathcal{E}}(\theta) \Im\left(r^{£} \theta\right) \wp_{0} d \theta d r, \\
& =\frac{£(\psi(t))^{(1+£ \S)(1-\beta)}}{\Gamma(\beta(1-£))} \mathfrak{T}\left(\zeta^{£} \sigma\right) \int_{\zeta}^{t} \int_{\sigma}^{\infty} \psi^{\prime}(\psi(t)-\psi(r))^{(1-£) \beta-1} r^{£-1} \theta M_{\mathcal{E}}(\theta) \Im\left(r^{£} \theta-\zeta^{£} \sigma\right) \wp_{0} d \theta d r .
\end{aligned}
$$

Moreover,

$$
\begin{aligned}
& \left\|\left(\mathfrak{F}^{1} y\right)(t)-\left(\mathfrak{F}_{\zeta, \sigma}^{1} y\right)(t)\right\| \\
& \leq \mathscr{K}(£, \beta)\left\|(\psi(t))^{(1+£ \S)(1-\beta)} \int_{0}^{t} \int_{0}^{\sigma} \psi^{\prime}(\psi(t)-\psi(r))^{\beta(1-£)-1} r^{£-1} \theta M_{£}(\theta) \Im\left(r^{£} \theta\right) \wp_{0} d \theta d r\right\| \\
& +\mathscr{K}(\psi(t))^{-£ \beta(1+\S)}\left\|\wp_{0}\right\| \int_{0}^{\zeta}(1-s)^{\beta(1-£)-1} r^{-£ \S-1} d r \int_{\eta}^{\infty} \theta^{-\S} M_{£}(\theta) d \theta \\
& \rightarrow 0, \text { as } \zeta \rightarrow 0, \sigma \rightarrow 0,
\end{aligned}
$$

where, $\mathscr{K}(£, \beta)=\frac{£}{\Gamma(\beta(1-£))}$.

Therefore, $\left.\mathcal{V}_{\zeta, \sigma}^{1}(t)=\left\{\mathfrak{F}_{\zeta, \sigma}^{1} y\right)(t), y \in \mathfrak{B}_{r}(\mathcal{J})\right\}$ are arbitrarily close to $\mathcal{V}^{1}(t)=\left\{\mathfrak{F}^{1} y\right)(t)$. Now we can present $\mathfrak{F}_{\zeta, \sigma}^{2}$ on $\mathfrak{B}_{r}(\mathcal{J})$ by

$$
\begin{aligned}
& \left(\mathfrak{F}_{\zeta, \sigma}^{2} y\right)(t)=£(\psi(t))^{(1+£ \S)(1-\beta)} \int_{0}^{t-\zeta} \int_{\sigma}^{\infty} \theta M_{\mathcal{E}}(\theta) \psi^{\prime}(\psi(t)-\psi(r))^{£-1} \Im\left(\psi^{\prime}(\psi(t)-\psi(r))^{£} \theta\right) \mathscr{E}(r, \wp(r)) d \theta d r \\
& +\sum_{0<t_{k}<t} \psi_{0}^{£, \beta}\left(\psi(t)-\psi\left(t_{k}\right)\right) \mathscr{I}_{k}\left(\wp\left(t_{k}^{-}\right)\right), \\
& =£(\psi(t))^{(1+£ \S)(1-\beta)} \mathfrak{T}\left(\zeta^{£} \sigma\right) \int_{0}^{t-\zeta} \int_{\sigma}^{\infty} \theta M_{£}(\theta) \psi^{\prime}(\psi(t)-\psi(r))^{£-1} \\
& \Im\left(\psi^{\prime}(\psi(t)-\psi(r))^{£} \theta-\zeta^{£} \sigma\right) \mathscr{E}(r, \wp(r)) d \theta d r+\sum_{0<t_{k}<t} \psi_{0}^{£, \beta}\left(\psi(t)-\psi\left(t_{k}\right)\right) \mathscr{I}_{k}\left(\wp\left(t_{k}^{-}\right)\right) .
\end{aligned}
$$

Hence

$$
\begin{aligned}
\left\|\left(\mathfrak{F}^{2} y\right)(t)-\left(\mathfrak{F}_{\zeta, \sigma}^{2} y\right)(t)\right\| & \leq \| £(\psi(t))^{(1+£ \S)(1-\beta)}\left(\int_{0}^{t} \int_{0}^{\sigma} \theta M £(\theta) \psi^{\prime}(\psi(t)-\psi(r))^{£-1}\right. \\
& \left.\Im\left((\psi(t)-\psi(r))^{£} \theta\right) \mathscr{E}(r, \wp(r)) d \theta d r+\sum_{0<t_{k}<t} \psi_{0}^{£, \beta}\left(\psi(t)-\psi\left(t_{k}\right)\right) \mathscr{I}_{k}\left(\wp\left(t_{k}^{-}\right)\right)\right) \| \\
& \rightarrow 0 \text { as } \zeta \rightarrow 0, \sigma \rightarrow 0 .
\end{aligned}
$$

Therefore, $\left.\mathcal{V}_{\zeta, \sigma}^{2}(t)=\left\{\mathfrak{F}_{\zeta, \sigma}^{2} y\right)(t), y \in \mathfrak{B}_{r}(\mathcal{J})\right\}$ are arbitrarily close to $\mathcal{V}^{2}(t)=\left\{\mathfrak{F}^{2} y\right)(t)$, $\left.y \in \mathfrak{B}_{r}(\mathcal{J})\right\}, t>0$. That is, $\left\{\mathfrak{F} y, y \in \mathfrak{B}_{r}(\mathcal{J})\right\}$ is relatively compact using the Arzela-Ascoli Theorem. Then $\wp^{*}$ is a mild solution of (1)-(3).

Hence by the Schauder fixed-point theorem, there exist a fixed point $y^{*} \in \mathfrak{B}_{r}(\mathcal{J})$ of $\mathfrak{F}$. Let $\wp^{*}(t)=t^{(1+£ \S)(\beta-1)} y^{*}(t)$. Then $\wp^{*}(t)$ is a mild solution of $(1)-(3)$. 


\section{5. $\Im(t)$ Is Noncompact}

We consider as follows:

Hypothesis 5 (H5). $\exists$ a constant $k>0$ fulfills the following criteria

$$
\aleph\left(\mathscr{E}\left(t, \mathfrak{E}_{1}, \mathfrak{E}_{2}\right)\right) \leq k \aleph\left(\mathfrak{E}_{1}, \mathfrak{E}_{2}\right) \text { for particularly } t \in[0, T] \text { and } \mathfrak{E}_{1}, \mathfrak{E}_{2} \subset Y .
$$

and $\mathfrak{E}_{1}, \mathfrak{E}_{2} \subset Y$.

Theorem 4. Let $-1<\S<0,0<\omega<\frac{\pi}{2}$ and $\mathfrak{A} \in \aleph_{\omega}^{\S}$. Assume that (H1)-(H5) are true. Then (1)-(3) offers a mild solution in $\mathfrak{B}_{r}^{Y}(J)$ for each $r$ and for every $u_{0} \in D(\mathscr{E} \theta)$ with $\theta>1+\S$.

Proof. Now we prove that $\mathfrak{F}$ is compact in $\mathfrak{B}_{r}(\mathcal{J})$.

For the set with a boundary $\mathbb{P}_{0} \subset \mathfrak{B}_{r}(\mathcal{J})$, set

$$
\mathfrak{F}^{(1)}\left(\mathbb{P}_{0}\right)=\mathfrak{F}\left(\mathbb{P}_{0}\right), \mathfrak{F}^{(n)}\left(\mathbb{P}_{0}\right)=\mathfrak{F}\left(\overline{c o}\left(\mathfrak{F}^{(n-1)}\left(\mathbb{P}_{0}\right)\right)\right), n=2,3, \cdots
$$

If $\mathfrak{F}$ is greater than 0 , since ([12] (2.2)-(2.4)), a subsequence $\left\{y_{n}^{(1)}\right\}_{n=1}^{\infty} \subset \mathbb{P}_{0}$ satisfying

$$
\begin{aligned}
\aleph\left(\mathfrak{F}^{(1)}\left(\mathbb{P}_{0}(t)\right)\right) & \leq 2 \aleph\left((\psi(t))^{(1+£ \S)(1-\beta)} \int_{0}^{t} \psi^{\prime}(\psi(t)-\psi(r))^{£-1} \mathfrak{Q}_{£}(\psi(t)-\psi(r))\right. \\
& \left.\mathscr{E}\left(r,\left\{r^{-(1+£ \S)(1-\beta)}\left(y_{n}^{(1)}(r)\right)\right\}_{n=1}^{\infty}\right) d r+\sum_{0<t_{k}<t} \omega_{k}\right) \\
& =4 \mathfrak{C}_{p} k(\psi(t))^{-£ \S}\left(\mathfrak{P}_{0}\right)\left(\frac{\Gamma(-£ \S) \Gamma((-£ \S+\beta(1+£ \S))}{\Gamma(-2 £ \S+\beta(1+£ \S))}+\sum_{0<t_{k}<t} \omega_{k}\right) .
\end{aligned}
$$

For $\mathfrak{F}$ is arbitrary,

$\aleph\left(\mathfrak{F}^{(1)}\left(\mathbb{P}_{0}(t)\right)\right) \leq 4 \mathfrak{C}_{p} k(\psi(t))^{-£ \S} \aleph\left(\mathbb{P}_{0}\right)\left(\frac{\Gamma(-£ \S) \Gamma((-£ \S+\beta(1+£ \S))}{\Gamma(-2 £ \S+\beta(1+£ \S))}+\sum_{0<t_{k}<t} \omega_{k}\right)$.

Now,

$$
\aleph\left(\mathfrak{F}^{(n)}\left(\mathbb{P}_{0}(t)\right)\right) \leq \frac{\left(4 \mathfrak{C}_{p} k\right)^{n}(\psi(t))^{-n £ \S} \Gamma^{n}(-£ \S) \Gamma(-£ \S+\beta(1+£ \S))}{\Gamma(-(n+1) £ \S+\beta(1+£ \S))} \aleph\left(\mathbb{P}_{0}\right), n \in \mathbb{N} .
$$

Changing $(n+1)$ by $(j+1) k$. We obtain

$$
\frac{\left(4 \mathfrak{C}_{p} k^{n} t^{-n £ \S} \Gamma^{n}(-£ \S) \Gamma(-£ \S+\beta(1+£ \S)\right.}{\Gamma(-(n+1) £ \S+\beta(1+£ \S))} \leq \frac{\left(4 \mathfrak{C}_{p} k\right)^{n_{0}} T^{-n_{0} £ \S} \Gamma^{n_{0}}(-£ \S) \Gamma(-£ \S+\beta(1+£ \S))}{\Gamma\left(-\left(n_{0}+1\right) £ \S+\beta(1+£ \S)\right)}=p<1 .
$$

Now

$$
\aleph\left(\mathfrak{F}^{\left(n_{0}\right)}\left(\mathbb{P}_{0}(t)\right)\right) \leq p \aleph\left(\mathbb{P}_{0}\right) .
$$

From boundedness and equicontinuity of $\mathfrak{F}^{\left(n_{0}\right)}\left(\mathbb{P}_{0}(t)\right)$ and by applying [12] (Proposition 2.2), we obtain

$$
\aleph\left(\mathfrak{F}^{\left(n_{0}\right)}\left(\mathbb{P}_{0}\right)\right)=\max _{t \in[0, T]} \aleph\left(\mathfrak{F}^{n_{0}}\left(\mathbb{P}_{0}(t)\right)\right) .
$$

Hence,

$$
\aleph\left(\mathfrak{F}^{n_{0}}\left(\mathbb{P}_{0}\right)\right) \leq p \aleph\left(\mathbb{P}_{0}\right)
$$


where $p<1$. Hence by the Schauder fixed-point theorem, we obtain a fixed point $y^{*} \in$ $\mathfrak{B}_{r}(\mathcal{J})$ of $\mathfrak{F}$. Let $\wp^{*}(t)=t^{(1+\mathcal{S})(\beta-1)} y^{*}(t)$. Then $\wp^{*}(t)$ is a mild solution of (1)-(3).

\section{Illustrate an Abstract Application}

We consider an abstract application via Hilfer fractional derivative system:

$$
\begin{aligned}
\mathfrak{D}_{0+}^{\alpha, \gamma ; \psi} \wp(t, \mathfrak{x})-\partial_{\mathfrak{x}}^{2} \wp(t, \mathfrak{x}) & =\mathscr{E}(t, \wp(t, \mathfrak{x})) t \in[0, T], \mathfrak{x} \in[0, \iota], \\
\wp(t, 0)=\wp(t, \iota) & =0 \text { on } t \in[0, T], \\
\mathcal{I}_{0+}^{(1-\alpha)(1-\gamma) ; \psi} \wp(0, \mathfrak{x}) & =\wp_{0}(\mathfrak{x}), \mathfrak{x} \in[0, a], \\
\left.\Delta \wp\right|_{t=1 / 2} & =\mathscr{I}_{1}\left(\wp\left(\frac{1}{2}^{-}\right)\right),
\end{aligned}
$$

in Banach space $\mathscr{Y}=C^{\alpha}([0, a])(0<\alpha<1)$, where $\alpha=\frac{1}{4}, \gamma=\frac{1}{2}, \mathscr{E}(t, \wp)=t^{-\frac{1}{5}} \cos ^{2} \wp$. Here, we can convert the above problem into system (1)-(3) in the abstract form as

$$
\begin{aligned}
\mathfrak{D}^{\alpha, \gamma ; \psi} \wp(t)+\mathscr{A} \wp(t) & =\mathscr{E}(t, \wp(t)) \quad t \in(0, T]=\mathcal{J}, \\
\left.\Delta \wp\right|_{t=t_{k}} & =\mathscr{I}_{k}\left(\wp\left(t_{k}^{-}\right)\right), k=1,2,3, \ldots, m, \\
I_{0+}^{(1-\alpha)(1-\gamma) ; \psi} \wp(0) & =\wp_{0} .
\end{aligned}
$$

Here $\mathscr{A}=-\partial_{x}^{2}$ with $\mathfrak{D}(\mathscr{A})=\left\{\wp \in C^{2+\alpha}([0, \iota])\right.$ therefore $\left.\wp(t, 0)=0=\wp(t, \iota)\right\}$. Since by [14] there $\exists$ constants $\delta, \mathfrak{F}>0$, implies $\mathscr{A}+\delta \in \odot_{\frac{\pi}{2}-\mathfrak{F}}^{\frac{\pi}{2}-1}(\mathscr{Y})$. To verify the compactness of semigroup $\Im(t)$, it is enough to prove that $\mathscr{R}\left(\alpha,-(\mathscr{A}+\delta)\right.$ is compact. We take $l(t)=t^{-\frac{1}{5}}$.

$$
\left.r=\sup _{[0, T]}\left(\psi_{0}(t)\right)^{(1+\alpha \beta)(1-\gamma)} \wp_{0} \|\right)+\frac{T^{\frac{17}{20} \Gamma\left(-\frac{\beta}{4}\right) \Gamma\left(\frac{4}{5}\right)}}{\Gamma\left(\frac{4}{5}-\frac{\beta}{4}\right)} .
$$

Then the Hypotheses (H1)-(H5) are satisfied. According to Theorem 3, problem (8) has a mild solution in $\mathfrak{B}_{r}^{\mathfrak{Y}}((0, T])$.

\section{Conclusions}

In this manuscript, using the Schauder fixed-point theorem, we investigated mild solutions for $\psi$-Hilfer fractional derivative differential equations involving jump conditions and a nearly sectorial operator when the related semigroup is compact and noncompact. Finally, an illustration of the key result is discussed. Our theorems ensure that the existence results are effective. One can extend the same problem to controllability problem for autonomous and non-autonomous systems. Additionally, we can study time-dependent and state-dependent controllability of the system (1)-(3) in the extended form. Numerical results of the system will be quite interesting.

Author Contributions: Formal analysis, K.K., D.N.C.; Investigation, P.K., D.N.C.; Methodology, P.K., D.N.C., D.S.R., P.S. All authors have read and agreed to the published version of the manuscript.

Funding: This research received no external funding.

Institutional Review Board Statement: Not applicable.

Informed Consent Statement: Not applicable.

Conflicts of Interest: The authors declare no conflict of interest.

\section{References}

1. Hilfer, R. Applications of Fractional Calculus in Physics; World Scientific: Singapore, 2000.

2. Hilfer, R. Experimental evidence for fractional time evolution in glass forming materials. Chem. Phys. 2002, 284, 399-408. [CrossRef] 
3. Ahmed, H.M.; El-Borai, M.M.; El-Owaidy, H.M.; Ghanem, A.S. Impulsive Hilfer fractional derivative differential equations. Adv. Differ. Equ. 2018, 2018, 226. [CrossRef]

4. Kamocki, R. A new representation formula for the Hilfer fractional derivative and its application. J. Comput. Appl. Math. 2016, 308, 39-45. [CrossRef]

5. Abdo, M.S.; Thabet, S.T.; Ahmad, B. The existence and Ulam-Hyers stability results for $\psi$-Hilfer fractional integrodifferential equations. J. Pseudo-Differ. Oper. Appl. 2021, 11, 1757-1780 [CrossRef]

6. Derbazi, C.; Baitiche, Z.; Benchohra, M.; Cabada, A. Initial value problem for nonlinear fractional differential equations with $\psi$-Caputo derivative via monotone iterative technique. Axioms 2020, 9, 57. [CrossRef]

7. Mali, A.D.; Kucche, K.D.; da Costa, S.J.V. On Coupled System of Nonlinear $\psi$-Hilfer Hybrid Fractional Differential Equations. arXiv 2021, arXiv:2012.02949.

8. Kucche, K.D.; Kharade, J.P.; Sousa, J. On the Nonlinear $\psi$-Hilfer Fractional Differential Equations. Comput. Appl. Math. 2019, 38, 1-25. [CrossRef]

9. Da Costa, S.J.V.; Kucche, K.D.; de Oliveira, E.C. Stability of $\psi$-Hilfer impulsive fractional differential equations. Appl. Math. Lett. 2019, 88, 73-80. [CrossRef]

10. Da Costa, S.J.V.; de Oliveira, E.C. A Gronwall inequality and the Cauchy-type problem by means of $\psi$-Hilfer operator. arXiv 2017, arXiv:1709.03634.

11. Da Costa Sousa, J.V.; de Oliveira, E.C. On the $\psi$-Hilfer fractional derivative. Commun. Nonlinear Sci. Numer. Simulat. 2018, 60, 72-91. [CrossRef]

12. Banas, J.; Goebel, K. Measures of Noncompactness in Banach Spaces; Lecture Notes in Pure and Applied Mathematics; Marcel Dekker: New York, NY, USA, 1980; Volume 60.

13. Gu, H.; Trujillo, J.J. Existence of mild solution for evolution equation with Hilfer fractional derivative. Appl. Math. Comput. 2015, 257, 344-354. [CrossRef]

14. Pazy, A. Semigroup of Linear Operators and Applications to Partial Differential Equations; Applied Mathematical Sciences; Springer: Berlin, Germany, 1983; Volume 44.

15. Zhou, Y.; Wang, J.; Zhang, L. Basic Theory of Fractional Differential Equations; World Scientific: Singapore, 2014. 